\title{
ALTERNATIVE INTERLEAVING SCHEMES FOR INTERLEAVED ORTHOGONAL FREQUENCY DIVISION MULTIPLEXING
}

\author{
Karthik Subramanian and K.V.S Hari \\ Department of Electrical Communication Engineering \\ Indian Institute of Science \\ Bangalore 560012, INDIA \\ 080-293-2745 \\ karthik@dsplab.ece.iisc.ernet.in, hari@ece.iisc.ernet.in
}

\begin{abstract}
We propose a matrix model for the Interleaved Orthogonal Frequency Division Multiplexing System (IOFDM) [3], [4], [5]. Using this model, we show that the interleaving scheme originally proposed for IOFDM is not unique in terms of preserving system complexity. We further show that a class of interleaving matrices yields IOFDM systems with the same complexity as the original IOFDM system. Furthermore, we show that the interleaving scheme does not affect the BER performance of the system as long as the interleaving matrix is unitary.
\end{abstract}

\section{INTRODUCTION}

Orthogonal frequency division multiplexing (OFDM)[1], [2] is an effective technique for data transmission over frequency selective channels. However, due to the redundancy introduced in the form of the zero-pad or the cyclic prefix, OFDM has a loss in code rate. Interleaved OFDM [3],[4],[5] is an OFDM system which offers an improvement in code rate without an increase in the FFT size used, by interleaving several data blocks using a specific scheme before the addition of the cyclic prefix/zero pad. IOFDM was proposed with a specific interleaving scheme. We propose a simplified matrix model for IOFDM and use this model to show that the interleaving scheme is not unique in terms of yielding an efficient receiver structure. We further state a result giving a class of interleaving schemes that yield IOFDM systems with the same complexity as the original IOFDM system. We also show that as long as the interleaving matrix is unitary, it does not affect the BER of the system.

\section{A BRIEF REVIEW OF IOFDM}

Figure (1) shows an IOFDM Transmitter. The information sequence $s(n)$ consists of complex numbers obtained by mapping a binary sequence to a constellation, and is blocked into a vector sequence $\underline{s}(n)$ of size $K \times 1^{1}$. Next, $P$ consecutive blocks of size $K \times 1$ are blocked to get a composite block of size $P K \times 1$. This block is pre-processed by multiplying the block elements by scalars, which is represented

0-7803-7651-X/03/\$17.00 ๑ 2003 1EEE

${ }^{1} \mathrm{~K}$ is the OFDM block size / FFT size, and $\mathrm{P}$ is the interleaving factor Hence the lOFDM block size is $P K \times 1$. by the $\mathbf{D}^{l}$ blocks ${ }^{2}, \mathbf{D}^{l}=\operatorname{diag}\left[1, W_{P K}, \ldots, W_{P K}^{K-1}\right], l \in$ $\{0,1, \ldots, P-1\}$. Each component $K \times 1$ block is then subjected to a $K$-point IDFT, as represented by the blocks $\mathrm{F}_{K}^{\mathcal{H}}$ and the composite $P K \times 1$ block is subjected to interleaving, and finally a zero pad of length $L$ equal to the maximum channel length is appended to the IOFDM block.The size of the block with zero-pad added is $M=P K+L$. This block sequence $\underline{x}(n)$ is now converted to serial and then fed to a single antenna for transmission across the channel.



Figure 1. IOFDM Transmitter

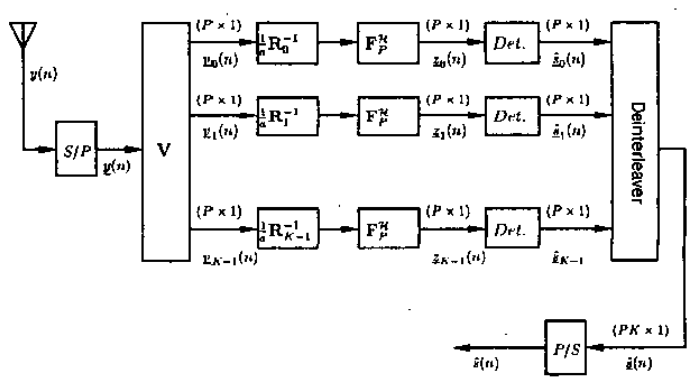

Figure 2. IOFDM Receiver

To illustrate the interleaving scheme, consider a sequence ${ }^{3}$ $\left[a_{0}, a_{1}, \ldots a_{K-1}, b_{0}, b_{1}, \ldots b_{K-1}\right]^{T}$. ( We have consid-

\footnotetext{
${ }^{2} W_{K}=e^{j \frac{\pi}{K}}$

${ }^{3} T$ stands for transpose. $\mathcal{H}$ is the Hermitian operator, an underline denotes a vector. $\mathbf{F}_{K}$ is a $K \times K$ DFT matrix.
} 
Communications-1/1̦47.

ered $P=2$ here ). The interleaved sequence is now $\left[a_{0}, b_{0}, a_{1}, b_{1}, \ldots, a_{K-1}, b_{K^{\prime}-1}\right]^{T}$. If we choose the interleaving factor $P$ to be 1, IOFDM reduces to the familiar OFDM system. At the receiving end, the serial sequence is blocked into blocks of size $P K \times 1$, and the zero pad is stripped from each block. The blocks are now subjected to a $P K$ point DFT. This is represented by the $\mathrm{V}$ matrix, where $\mathbf{V}=\mathbf{F}_{{ }^{\prime}} \mathbf{R}_{Z \nu}, \mathbf{R}_{\not P}$ representing the zero-pad removal. Equalization is done by simple scalar division as represented by the $\mathbf{R}_{\boldsymbol{l}}$ blocks, given by

$\mathbf{R}_{l}=\operatorname{diag}\left[H\left(e^{j \frac{2 \pi l}{P K}}\right), H\left(e^{j \frac{3 \pi(K+l)}{P K}}\right), \ldots, H\left(e^{j \frac{2 \pi i(P-1) K+l)}{P K}}\right)\right]$, $\forall l \in\{0, K-1\}$, where $H\left(e^{j \frac{2 \pi l}{P K}}\right)$ is the channel frequency response evaluated at the $l^{t h}$ subcartier frequency. The block sequence is now subjected to a $P$ point IDFT, de-interleaved and fed to the detector. This yields an estimate of the information sequence $s(n)$. A complete exposition of IOFDM can be found in [4].

\section{MATRIX MODEL FOR IOFDM}

Figure (3) shows the matrix models for the IOFDM transmitter and receiver. $s(n)$ is the complex modulated information sequence, which is blocked into a $P K \times 1$ vector sequence, $\underline{r}(n) . \mathrm{G}^{-1}$ is the pre-processor, $\mathrm{C}_{P K}$ is a $P K \times P K$ block IDFT comprising $K \times K$ IDFT blocks ${ }^{4}$. $\mathrm{L}_{P K}$ is the interleaving matrix, and $\mathbf{T}_{Z P}=\left[\begin{array}{ll}\mathbf{I}_{P K}^{T} & \mathbf{0}_{L \times P K}^{T}\end{array}\right]^{T}$ is the zero-pad addition matrix. Thus we have at the output of the zero-pad block:

$$
\begin{aligned}
\underline{x}(n) & =\mathbf{T}_{Z P} \mathbf{L}_{P K} \mathbf{C}_{P K} \underline{g}(n) \\
\underline{g}(n) & =\mathbf{G}^{-1} \underline{r}(n) \\
\underline{r}(n) & =[\underline{s}(n P), \ldots, \underline{s}(n P+P-1)]^{T} \\
& =[s(K P), \ldots, s(6)+P K-1)]^{T}
\end{aligned}
$$
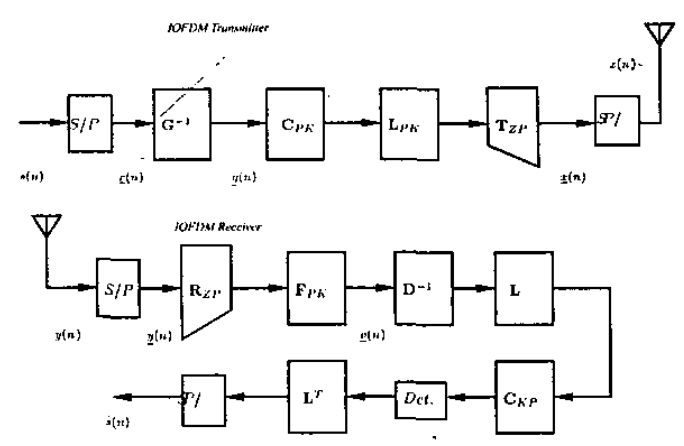

Figure 3. Matrix Model for IOFDM

\footnotetext{
${ }^{4} \mathbf{C}_{l^{\prime} K}=\operatorname{diag}\left(\mathbf{F}_{K}^{H}, \mathbf{F}_{K}^{H}, \ldots, \mathbf{F}_{K}^{H}\right)_{P K \times P K}$
}

We define $\mathrm{L}_{P^{\prime} K^{\prime}}$ as

$$
\mathbf{L}_{P K^{\prime}}=\left[\begin{array}{cccc}
\delta_{0,0} & \delta_{1,0} & \ldots & \delta_{P-1,0} \\
\delta_{0,1} & \delta_{1,1} & \ldots & \delta_{P-1,1} \\
\vdots & \vdots & \vdots & \vdots \\
\delta_{0, K-1} & \delta_{1, K-1} & \ldots & \delta_{P-1, K-1}
\end{array}\right]
$$

Where each $\delta_{i, j}$ is a $P \times K$ matrix of zeros, with a 1 at the $(i, j)^{t h}$ entry.

$$
\delta_{i, j}(m,)= \begin{cases}0, & i \neq m \dot{m}, \quad \neq n \\ 1, & i=m \dot{m}, \quad=n\end{cases}
$$

$\mathbf{L}_{\mu_{K}}$ thus defined is the interleaving matrix corresponding to the original IOFDM interleaving scheme. The signal after passing through the linearly convolutive channel with impulse-response $h(n)$ and being corrupted with additive white Gaussian noise, is represented as

$$
\underline{y}(n)=\mathbf{H} \underline{x}(n)+\underline{\eta}(n)
$$

$\mathbf{H}$ is the channel-convolution matrix [1], $\underline{\eta(n)}$ is the noise component and $y(n)$ is the signal at the receiver's front-end. The operations performed on the received signal can be written as follows:

$$
\begin{aligned}
\underline{v}(n) & =\mathbf{F}_{P K} \mathbf{R}_{Z P} \underline{y}(n) \\
\underline{v}(n) & =\mathbf{F}_{P K} \mathbf{R}_{Z P} \mathbf{H} \mathbf{T}_{Z P} \mathbf{L}_{P K} \cdot \mathbf{C}_{P K} \underline{\underline{g}}(n)+\underline{\eta^{\prime}(n)} \\
\underline{\eta^{\prime}}(n) & =\mathbf{F}_{P K} \mathbf{R}_{Z P} \underline{\eta}(n)
\end{aligned}
$$

Where $\mathbf{R}_{Z P}=\left[\begin{array}{ll}\mathbf{I}_{\mathbf{P K}} & \mathbf{0}_{\mathbf{P K} \times \mathbf{L}}\end{array}\right]$ is the zero-pad removal matrix, and $\mathbf{F}_{P K}$ is a $P K$-point DFT matrix. We know that the product $\tilde{\mathbf{H}}=\mathbf{R}_{\mathbf{Z P}} \mathbf{H} \mathbf{T}_{\mathbf{Z P}}$. is a circulant matrix [1], and can be factorized as $\tilde{\mathbf{H}}=\mathbf{F}_{P K}^{H I} \mathbf{D} \mathbf{F}_{P K}[6]$, and hence we have.

$$
\underline{v}(n)=\mathbf{D} \mathbf{F}_{P K} \mathrm{~L}_{P K} \mathbf{C}_{P K} \underline{g}(n)+\underline{\eta}^{\prime}(n)
$$

$\mathbf{D}=\operatorname{dictg}\left[H(1) H\left(e^{j \frac{2 \pi 1}{P K}}\right), \ldots, H\left(e^{j \frac{2 \pi(p K-1)}{p K}}\right)\right]$ is a diagonal matrix and is easily inverted by scalar division. The issue now is to efficiently invert the product $\mathbf{A}=\mathbf{F}_{P K} \mathrm{~L}_{P K} \mathbf{C}_{P^{\prime} K}$, which has the following structure:

$\mathbf{A}=$

$$
K\left[\begin{array}{cccc}
D_{0} & D_{1} & \ldots & D_{P-1} \\
D_{0} & W_{P K}^{K} D_{1} & \ldots & W_{P K}^{(P-1) K} D_{P-1} \\
& & \vdots & \\
D_{0} & W_{P K}^{(P-1) * K} D_{1} & \ldots & W_{P K}^{(P-1)^{2} K} D_{P-1}
\end{array}\right]
$$

Where $D_{l}:=\operatorname{diag}\left(1, W_{P K}^{l}, \ldots, W_{P K}^{(K-1) \times l}\right)$. We make use of the following fact to factorize $\mathbf{A}$ into an easily invertible product: The matrix $\mathbf{Z}=$ 


\section{TENCON $2003 / 148$}

$$
\left[\begin{array}{cccc}
\mathbf{I}_{K} & \mathbf{I}_{K} & \ldots & \mathbf{I}_{K} \\
\mathbf{I}_{K} & W_{P} \mathbf{I}_{K} & \ldots & W_{P}^{P-1} \mathbf{I}_{K} \\
& & \vdots & \\
\mathbf{I}_{K} & W_{P}^{P-1} \mathbf{I}_{K} & \ldots & W_{P}^{(P-1)^{2}} \mathbf{I}_{K}
\end{array}\right] \text { can be }
$$

factorized as $\mathbf{Z}=\mathbf{L}^{T} \mathbf{C}_{K P}^{H} \mathbf{L}$, where $\mathbf{L}$ is the original interleave inatrix defined in (4), and $\mathbf{C}_{K P}^{H}$ is a $P K \times P K$ block DFT, comprising $K$ blocks of $P \times P$ DFT's. That is, the matrix $\mathbf{Z}$ is merely a re-indexed version of the block DFT matrix $\mathbf{C}_{K^{\prime} P}^{H}$.

Defining $\mathbf{G}=\operatorname{diag}\left[D_{0}, D_{1}, \ldots, D_{P-1}\right]$, we see that $\mathbf{A}=$ $\mathbf{Z ~ G}=\mathbf{L}^{T} \mathbf{C}_{K P}^{I}, \mathbf{L} \mathbf{G}$. In other words,

$$
\begin{array}{r}
\mathbf{F}_{P K} \mathbf{L}_{P K} \mathbf{C}_{P K}=\mathbf{L}^{t} \mathbf{C}_{K P}^{\mathcal{H}} \mathbf{L} \mathbf{G} \\
\underline{v}(n)=\mathbf{D} \mathbf{L}^{T} \mathbf{C}_{K P}^{H} \mathbf{L} \mathbf{G} \underline{r}(n)+\underline{\eta}^{\prime}(n)
\end{array}
$$

Since $\underline{r}(n)=\mathbf{G}^{-1} g(n)$, the vector information sequence $g(n)$ can be recovered from $\underline{v}(n)$ in the following way, that yields an efficient receiver structure:

$$
\underline{\hat{g}}(n)=\mathbf{L}^{T} \mathbf{C}_{K P}\left(\mathbf{D L}^{T}\right)^{-1} \underline{v}(n)
$$

It can be seen that the matrix equation above corresponds to the IOFDM receiver structure shown in the previous section.

\section{INTERLEAVING SCHEMES PRESERVING SYSTEM COMPLEXITY}

From the previous section, we see that at the receiver:

$$
\underline{v}(n)=\mathbf{F}_{P K} \mathbf{L}_{P K} \mathbf{C}_{P K} \underline{s}(n)+\mathbf{D}^{-1} \mathbf{F}_{P K} \mathbf{R}_{Z P} \underline{\eta}(n)
$$

The signal $\underline{s}(n)$ can be recovered from this equation as long as the matrix $\mathrm{L}_{P K}$ is invertible. In other words, the interleaving scheme is not unique. Permutation matrices are attractive because of their low complexity of implementation. For any choice of $L_{P K}$ that is invertible, we can always find a pre-processing matrix $\mathbf{G}^{-1}$ that will ensure the block-DFT structure at the receiver. For the IOFDM system, $\mathbf{G}^{-1}$ is a diagonal matrix, resulting in $P K$ complex multiplications. In the worst case, a full preprocessing matrix $G^{-1}$ can have a complexity of $(P K)^{2}$ multiplications, which is a considerable increase in complexity. Hence this motivates us to look for interleaving matrices $\mathbf{L}_{P K}$ that result in a pre-processing matrix $\mathbf{G}^{-1}$ with low complexity of multiplication. A diagonal or an antidiagonal $\mathbf{G}^{-1}$ would be a good low-complexity solution, since this would result in only $P K$ multiplications for the preprocessing operation. However, an antidiagonal $\mathrm{G}^{-1}$ cannot be used in the current lOFDM framework, since the precoding matrix $\mathbf{C}$ is a (forward) block-diagonal. Hence we concentrate on interleaving matrices that result in a diagonal pre-processing matrix. We immediately notice that the use of the original interleaving matrix $\mathbf{L}_{P K}$ as defined in equation (3.3) results in a pre-processing matrix $\mathbf{G}^{-1}$ that is diagonal.

We state our question as What is the class of matrices $\mathrm{L}_{P K}$ that results in a diagonal pre-processing matrix $\mathrm{G}^{-1}$ for the IOFDM system?.
We know that

$$
\mathbf{F}_{P K} \mathbf{L}_{P K} \mathbf{C}_{P K} \mathbf{G}^{-1}=\mathbf{L}^{Y} \mathbf{C}_{K^{\prime} P}^{\mathcal{H}} \mathbf{L}
$$

This equation is now re-written as

$$
\mathbf{L}_{P K}=\mathbf{F}_{P K}^{\mathcal{H}} \mathbf{L}^{T} \mathbf{C}_{K P}^{\mathcal{H}} \mathbf{L} \mathbf{G}^{-1} \mathbf{C}_{P K}^{\mathcal{H}}
$$

We want to find the class of matrices $\mathbf{L}_{P K}$ such that $\mathbf{G}$ in the equation above is diagonal. Now, consider the product $\mathbf{F}_{P K}^{\mathcal{H}} \mathbf{L}_{P K}^{T} \mathbf{C}_{K P}^{\mathcal{H}}$. It can be seen that this product can be written as

$$
\mathbf{F}_{P K}^{\mathcal{H}} \mathbf{L}_{P K^{\prime}}^{T} \mathbf{C}_{K P}^{\mathcal{H}}=\mathbf{L}_{P K} \mathbf{C}_{P K} \mathbf{J} \mathbf{L}_{P K}^{T}
$$

Where, $\mathbf{J}$ is a diagonal matrix given by

$$
\mathbf{J}=\left[\begin{array}{cccc}
J^{0} & \mathbf{0} & \cdots & \mathbf{0} \\
\mathbf{0} & J^{1} & \cdots & \mathbf{0} \\
\vdots & \vdots & \ddots & \vdots \\
\mathbf{0} & \mathbf{0} & \ldots & J^{P-1}
\end{array}\right]
$$

and $J$ is $J=1\left[, W_{P K}^{-1}, W_{P K}^{-2}, \ldots, W_{P K}^{(K-1)}\right]^{T}$.

Inserting this into equation (3.15), we get

$$
\begin{aligned}
\mathbf{L}_{P K} & =\mathbf{L} \mathbf{C}_{P K} \mathbf{J} \mathbf{L}^{T} \mathbf{L} \mathbf{G} \mathbf{C}_{P K^{\prime}}^{\mathcal{H}} \\
& =\mathbf{L} \mathbf{C}_{P K} \mathbf{J} \mathbf{G} \mathbf{C}_{P K}^{\mathcal{H}}=\mathbf{L} \mathbf{C}_{P K} \mathbf{D}^{\prime} \mathbf{C}_{P K}^{\mathcal{H}}
\end{aligned}
$$

The matrices $\mathbf{J}$ and $\mathbf{G}$ are both diagonal, and hence their product $\mathbf{D}^{\prime}=\mathbf{J} \mathbf{G}$ is diagonal as well. $\mathbf{L}$ is the original interleaving matrix used in the IOFDM system [4].

Result: -To get a diagonal pre-processing matrix, the interleaving matrix $\mathrm{L}_{P K}$ must be of the form

$\mathbf{L}_{P K}=\mathbf{L} \mathbf{C}_{P K}\left[\begin{array}{cccc}\mathbf{D}^{\prime}{ }_{0} & \mathbf{0} & \ldots & \mathbf{0} \\ \mathbf{0} & \mathbf{D}^{\prime}{ }_{1} & \ldots & \mathbf{0} \\ \vdots & \vdots & \ddots & \vdots \\ \mathbf{0} & \mathbf{0} & \ldots & \mathbf{D}_{P-1}^{\prime}\end{array}\right] \mathbf{C}_{P K}^{\mathcal{H}}$

Where the matrices $\mathbf{D}^{\prime}{ }_{l}, l \in\{0,1, \ldots,(P-1)\}$ are diagonal matrices. In other words, the interleaving matrix should be 


\section{Communications-1/149}

a permuted $P K \times P K$ block diagonal matrix, with each of the $K \times K$ diagonal blocks being a circulant matrix. Such an interleaving matrix will yield an IOFDM. system with the same complexity as the original IOFDM system.

Remarks

1. The factorization $\mathbf{F}_{P K} \mathbf{L}_{P K} \mathbf{C}_{P K} \mathbf{G}^{-1}=\mathbf{L}^{T} \mathbf{C}_{K P}^{\mathcal{H}} \mathbf{L}$ is not unique. The factorization is valid for P-circular shifted versions of the matrix $\mathbf{L}$. However this does not affect the validity of the result in equation (3.18) in any way. This is because, $\mathbf{L}_{1}^{T} \mathbf{C}_{K P}^{\mathcal{H}} \mathbf{L}_{1}=\mathbf{L}_{2}^{T} \mathbf{C}_{K P}^{\mathcal{H}} \mathbf{L}_{2}$, for all $\mathbf{L}_{1}$ and $\mathbf{L}_{2}$ which are $\mathbf{P}$-circular shifted versions of $\mathbf{L}$. The equality is due to the block-diagonal symmetric structure of $\mathbf{C}_{K P}^{\mathcal{H}}$

2. The choice of interleaving scheme does not affect the performance of the system in any way. In the next section, we see that the BER of the Interleaved OFDM system is independent of the interleaving scheme used. This is borne out by simulations as well.

3. We see that for $\mathbf{L}_{P K}$ to be a permutation matrix, each of the constituent blocks of the block diagonal matrix $\mathbf{C}_{P K} \mathbf{D}^{\prime} \mathbf{C}_{P K}^{\mathcal{H}}$ must be a (circulant) permutation matrix. i.e, the elements of each diagonal block $\mathrm{D}_{l}, l \in\{0,1, \ldots, K-1\}$ should be the $K^{\text {th }}$ roots of unity, in some order. (This is because the eigenvalues of a circular-shift permutation matrix of order $K$ are the $K^{\text {th }}$ roots of unity in some order.)

\section{PROBABILITY OF ERROR FOR IOFDM}

We define $P_{\text {ber }, c}\left(\frac{E_{b}}{N_{0}}\right)$ to be the Probability of Error expression for the signal constellation $c$ at the SNR $\frac{E_{b}}{N_{0}}, E_{b}$ being the energy per bit, and $N_{0}$ being the noise power spectral density in Watts $/ \mathrm{Hz}$. We know that the Probability of Error of a conventional OFDM system using $K$ subcarriers can be derived as $P_{\text {elew }}=\frac{1}{K} \sum_{t=0}^{K-1} P_{b e r, c}\left(\frac{\left|H_{t}\right|^{2} E_{b}}{\left(1+\frac{L}{K}\right) N_{0}}\right)$ [7], with $P_{\text {ber, }}$ as defined above. For the sake of convenience, we assume in this section that in the IOFDM system, the received vector is processed and ML detected only after the de-interleaving at the receiver. In other words, the positions of the ML detector and the de-interleaver are interchanged. This does not affect the performance of the system, as we shall see. The received vector after processing now becomes

$$
\underline{\hat{s}}(n)=\underline{s}(n)+\mathbf{L}^{T} \mathbf{C}_{K P}^{\mathcal{H}} \mathbf{L} \mathbf{D}^{-1} \mathbf{F}_{P K} \mathbf{R}_{Z P} \underline{w}(n)
$$

It can be seen from the equation above that the energy per bit is unchanged at the receiver. However, the noise energy at the receiver is no longer $N_{0}$. The noise vector under consideration is $\mathbf{L}^{T} \mathbf{C}_{K P}^{\mathcal{H}} \mathbf{L} \mathbf{D}^{-1} \mathbf{F}_{P K} \mathbf{R}_{Z P} \underline{w}(n)$. We analyse the noise vector for both the cyclic prefix and the zero-padding cases.

\section{IOFDM with cyclic prefix}

Let $p$ be the number of bits per symbol in the constellation used. Then the total energy in the noise vector $\underline{w}(n)$ that is added to the signal at the receiver front-end is given by $p(P K+L) \mathrm{N}_{0} W_{s}$ where $W_{s}$ is the bandwidth occupied by each symbol. The noise vector at the output of the deinterleaver is given by

$$
\underline{\eta}(n)=\mathbf{L}^{T} \mathbf{C}_{K P}^{\mathcal{H}} \mathbf{L} \mathbf{D}^{-1} \mathbf{F}_{P K} \mathbf{R}_{C P} \underline{w}(n)
$$

where the cyclic-prefix removal matrix $\mathbf{R}_{C P}$ is given by $\mathbf{R}_{C P}=\left[\mathbf{0}_{P K \times I}^{T} \mathbf{I}_{P K \times P K}^{T}\right]^{T}$. The total energy in the vector $\mathbf{R}_{C P} \underline{w}(n), E_{w}$ is given by

$$
E_{w}=\mathscr{E}\left(\left(\mathbf{R}_{C P} \underline{w}(n)\right)^{\mathcal{H}}\left(\mathbf{R}_{C P} \underline{w}(n)\right)\right)
$$

Since the matrix $\mathbf{R}_{C P}$ is unitary, the expression on the RHS above becomes

$\mathscr{E}\left((\underline{w}(n))^{\mathcal{H}}(\underline{w}(n))\right)=p(P K+L) \mathbf{N}_{0} W_{s}$, which is the same as the energy in the vector before the removal of the cyclic prefix. However, the average noise PSD has now changed, since the number of symbols in the vector has become $P K$ from $P K+L$. The noise PSD in each subcarrier is given by $\mathrm{N}_{0} \frac{P K+L}{P K}$. All matrices in the matrix product in the noise vector under consideration are unitary, except for the diagonal matrix $\mathbf{D}^{-1}$. This vector represents equalization by scalar division for each subcarrier. Hence, the noise PSD after equalization in each subcarrier is given by $R_{\eta \eta}(l)=\mathrm{N}_{0} \frac{P K+L}{\left[H_{0}\right]^{2} P K}$. The average number of bad bits per received vector of $P K$ symbols (after processing at the receiver) is given by the sum of the bad bits in each subcarrier. Hence the total number of bad bits per block of $P K$ symbols is given by $\sum_{l=0}^{(P K-1)} P_{b e r, c}\left(\frac{E_{b}\left|H_{l}\right|^{2} P K}{(P K+L) N_{0}}\right)$. Thus we obtain an expression for the probability of error (averaged over several blocks of $P K$ symbols each) as:

$$
P_{e}=\frac{1}{P K} \sum_{l=0}^{P K-1} P_{b e r, c}\left(\frac{E_{b}\left|H_{l}\right|^{2} P K}{(P K+L) \mathbf{N}_{0}}\right)
$$

\section{IOFDM with Zero Padding}

We now examine the probability of error for an IOFDM system using zero-padding. The noise vector under consideration now becomes $\underline{\eta}(n)=\mathbf{L}^{T} \mathbf{C}_{K P} \mathbf{L} \mathbf{D}^{-1} \mathbf{F}_{P K} \mathbf{R}_{Z P} \underline{w}(n)$, where $\mathbf{R}_{Z P}$, the overlap-add matrix is given by $\mathbf{R}_{Z P}=$ $\left[\begin{array}{lll}\mathbf{I}_{P K} & \mathbf{I}_{Z P}\end{array}\right]$ where $\mathbf{I}_{Z P}$ is a matrix comprising the first $L$ columns of the identity matrix $I_{P K}$. The overlap-add matrix $\mathbf{R}_{Z P}$ is not unitary. The average energy in the vector $\mathbf{R}_{Z P} \underline{w}(n)$ is then given by $\mathscr{E}\left(\left(\mathbf{R}_{Z P} \underline{w}\right)^{\mathcal{H}}\left(\mathbf{R}_{Z P} \underline{w}\right)\right)$. Defining $\underline{w}=\left[w_{0} w_{1} \ldots w_{P K+L-1}\right]^{T}$, we see that the average energy in the vector $\mathbf{R}_{Z P} \underline{w}$ is given by

$$
\begin{aligned}
E\left(\mathbf{R}_{Z P} \underline{w}\right) & =\mathscr{E}\left(\left(\mathbf{R}_{Z P} \underline{w}\right)^{\mathcal{H}}\left(\mathbf{R}_{Z P} \underline{w}\right)\right) \\
& =p(P K+L) \mathbf{N}_{0} W_{s}
\end{aligned}
$$

The expectation of the cross terms is zero because the $w_{l}^{\prime} s$ are uncorrelated. As in the earlier section, the noise PSD in each subcarrier after equalization becomes $R_{\eta \eta}(l)=\mathrm{N}_{0} \frac{P K+L}{\left|H_{1}\right|^{2} P K}$. 
TENCON 2003/150

The probability of error for the IOFDM system with zero padding is then given by

$$
P_{e}=\frac{1}{P K} \sum_{l=0}^{P K-1} P_{b e r, c}\left(\frac{E_{b}\left|H_{l}\right|^{2} P K}{(P K+L) \mathbf{N}_{0}}\right)
$$

This expression is the same as that for the $P_{e}$ of an IOFDM system using the cyclic prefix.

Effect of the Interleaving Scheme on $P_{e}$ :

We see that the Probability of error of the LOFDM system is not affected by the choice of the "interleaving" matrix, as long as the matrix is unitary. Since any permutation matrix is unitary, the stacked data vectors at the transmitter can be interleaved in any fashion without affecting the bit-error ratio of the IOFDM system. This is borne out by the simulation results as well. If the "interleaving" matrix chosen is not unitary, it will affect the effective signal-to-noise ratio $\left(\frac{E_{b}}{N_{0}}\right)$, and hence the Probability of error as well.

\section{Comparison of $P_{e}$ between IOFDM and OFDM:}

The probability of error for OFDM and IOFDM are given below (assuming that we know the exact $P_{e}$ of the constellation used.)

$$
\begin{array}{r}
P_{e D N E}=\frac{1}{K} \sum_{l=0}^{K-1} P_{b e r, c}\left(\frac{\left|H_{l}\right|^{2} E_{b}}{\left(+\frac{L}{K}\right) \mathbf{N}_{0}}\right) \\
P_{e, \text { DNA }}=\frac{1}{P K} \sum_{l=0}^{P K-1} P_{b e r, c}\left(\frac{E_{b}\left|H_{l}\right|^{2}}{\left( \pm \frac{L}{P K}\right) \mathbf{N}_{0}}\right)
\end{array}
$$

We see that the probability of error of an IOFDM system with $K$ subcarriers and an interleaving factor of $P$ is exactly the same as that of an OFDM system with $P K$ subcarriers. Furthermore, for $K \searrow_{D}$, the $P_{e}^{\prime} s$ are approximately the same for both OFDM and IOFDM systems with $K$ subcarriers. So for large $P K$ (with $K \searrow>$ ), the probability of error of an IOFDM system is very close to that of an OFDM system.

\section{SimUlation Results}

In figure (4) we see the results of a comparison of the BER's of OFDM, IOFDM with the original interleaving scheme, and IOFDM with two different interleaving schemes. In the first scheme, no interleaving is done and the blocks are merely stacked. In the second one, a randomly generated permutation matrix was used for the interleaving scheme. The simulations were performed for the GSM HT channel.

\section{CONCLUSIONS}

It has been shown that a class of interleaving schemes exist for IOFDM which yield systems with the same complexity as the original IOFDM system. Furthermore, it has been shown

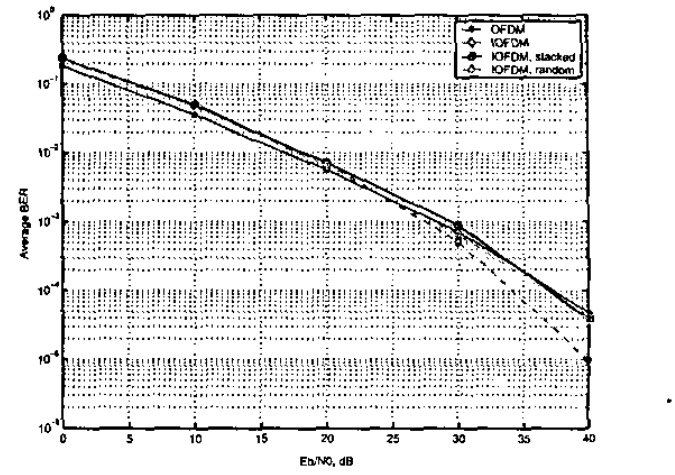

Figure 4. BER comparison, IOFDM with different interleave schemes

that the choice of the interleaving scheme does not affect the system performance as long as the interleaving matrix is unitary. Simulation results have been presented to bear out the same.

\section{REFERENCES}

[1] Zhengdao Wang, Georgios B. Giannakis, "Wireless Multicarrier Communications - where Fourier meets Shannon", IEEE Signal Processing Magazine, 29-48, May 2000.

[2] S. B Weinstein, Paul M. Ebert, "Data Transmission by Frequency-Division Multiplexing Using the Discrete Fourier Transform", IEEE Transactions on Communication Technology, COM-19, 628-634, October 1971.

[3] V.G.S Prasad, K.V.S Hari, "Interleaved Orthogonal Frequency Division Multiplexing System" in Proc. ICASSP 2002, Orlando, FL, May 2002, III.2745-2748, 2002.

[4] V.G.S Prasad, Multiple-Input Multiple-Output Techniques for Interleaved Orthogonal Frequency Division Multiplexing, Master's Thesis, Department of Electrical Communication Engineering, Indian Institute of Science, January 2002.

[5] V.G.S Prasad, K.V.S Hari, "Interleaved Orthogonal Frequency Division Multiplexing (IOFDM) System" IEEE Transactions on Signal Processing, To Appear.

[6] Gene H. Golub, Charles van Loan, Matrix Computations, 3rd ed. Baltimore, MD: Johns Hopkins University Press, 1996.

[7] Xiang-Gen Xia, "Precoded and Vector OFDM robust to Channel Spectral Nulls", IEEE Transactions on Communications, vol. 49, no. 8 , pp. 1363-1374. August 2001. 